\title{
Enfermedad incurable en la España del siglo XIX: el Hospital para Hombres Incurables Nuestra Señora del Carmen
}

\author{
Juan Manuel Zaragoza (*) \\ (*) Instituto de Filosofía. Centro de Ciencias Humanas y Sociales CSIC, Madrid. \\ jm.zaragozabernal@gmail.com
}

Dynamis

[0211-9536] 2012; $32(1): 141-163$
Fecha de recepción: 24 de febrero de 2011

Fecha de aceptación: 25 de noviembre de 2011

SUMARIO: 1.-Introducción. 2.-La reforma de la beneficencia. 3.-El Hospital de Hombres Incurables Nuestra Señora del Carmen. 3.1.-Reglamentos para la gestión de los Hospitales de Incurables. 3.2.-Peticiones de ingreso. 3.3.-Enfermedades y remedios. 3.4.-Recetas y cataplasmas. 4.-Conclusiones.

RESUMEN: El presente artículo analiza el proceso de asunción, por parte del Estado, de la asistencia médica de los ciudadanos «con necesidades permanentes». Dicha definición incluía a los enfermos incurables, a los enfermos crónicos y, también, a los ancianos. Este hecho coincide con la reforma de la Sanidad llevada a cabo por los diversos gobiernos liberales durante el reinado de Isabel II, encaminada a conseguir la creación y consolidación de una estructura sanitaria que, gradualmente, se hiciese cargo de las responsabilidades asumidas hasta ese momento por las fundaciones religiosas. La creación de dichas instituciones fue el resultado de los movimientos migratorios iniciados alrededor de 1830 e intensificados a partir de la década de 1850 , relacionados con el incipiente proceso de industrialización, y que habrían privado a los individuos de sus antiguas redes asistenciales: la familia y la comunidad más cercana. Este proceso, que se repite en otros países como Alemania o Inglaterra, supuso importantes cambios en diversos aspectos de la asistencia sanitaria española, no siendo el menor de ellos el impacto en la vida de los internados, cuyos últimos días pasaron a estar estrictamente controlados y medicalizados. El principal objetivo de este artículo es, precisamente, tratar de identificar los cambios producidos en la experiencia de enfermar de estos pacientes, a través del estudio de caso del Hospital para Hombres Incurables Nuestra Señora del Carmen, de Madrid, una de esas instituciones creadas para la atención de «necesidades permanentes» y, por tanto, bajo el control directo de la Administración General del Estado.

PALABRAS CLAVE: Beneficencia, Estado, incurables, experiencia del paciente, Madrid, España, siglo XIX.

KEY WORDS: Charity, state, incurables, patients' experience, Madrid, Spain, 19th century. 


\section{Introducción $(*)$}

Uno de los problemas a los que debe hacer frente toda forma de organización social es qué hacer con aquellos de sus miembros que, por motivos varios, no se encuentran en las condiciones óptimas para sustentarse a sí mismos. Las redes familiares y la beneficencia local fueron, en España y durante mucho tiempo, las responsables de asumir esta tarea. Estas redes desaparecieron a lo largo del siglo XIX debido a la elevada movilidad de la población que se trasladaba de sus lugares de origen a las grandes capitales donde se concentraba la industria, así como por las diversas desamortizaciones que, a lo largo del siglo, hicieron públicas las propiedades que habían sido las fuentes de ingreso principales de instituciones de beneficencia vinculadas a la Iglesia, junto a la abolición, por parte del gobierno liberal, de los cuerpos intermedios $^{1}$. Se creó, con esta desaparición, un vacío asistencial que se manifestaba con más crudeza en las clases más desfavorecidas de la sociedad. Con la llegada al poder de los liberales en 1833, y más concretamente con los gobiernos moderados que se sucedieron a partir de 1843, un nuevo concepto de beneficencia, basado en la moral católica y entendida como una virtud pública, llevó al Estado a considerar parte de sus obligaciones el cuidado de estos miembros desfavorecidos de la sociedad, creando, para ello, instituciones específicas que se hicieron cargo de esta tarea.

En el caso que nos ocupa, los hospitales de incurables, varias fueron las consecuencias de este cambio. Las primeras de orden teórico, ya que se debía encontrar una definición de «enfermedad incurable» que funcionara teórica pero también prácticamente: ya que no se podían atender las necesidades de todos los enfermos, sino tan sólo de aquellos que realmente no tuvieran acceso a otro medio de subsistencia, se debieron implementar requisitos de acceso que, como hemos defendido en artículos anteriores, pasaron a formar parte del concepto médico de «enfermedad incurable»a

(*) El presente trabajo se ha podido realizar gracias a una beca para la Formación de Investigadores, modalidad predoctoral, del Departamento de Educación, Universidades e Investigación del Gobierno Vasco, ref. BFI07.33, dentro de los proyectos de investigación «Epistemología Histórica; estilos emocionales en los siglos XIX y XX» (FFI2010-20876), financiado por el Ministerio de Ciencia e Innovación, y «CREP-CM-Estudios de Ciencia y Cultura: Culturas, Espacios, Representaciones y Prácticas», financiado por la Comunidad de Madrid.

1. Carasa Soto, Pedro. El sistema hospitalario español en el siglo XIX. De la asistencia benéfica al modelo sanitario actual. Valladolid: Universidad de Valladolid-Caja de Ahorros y Monte de Piedad de Salamanca; 1985, p. 45-46. 
nivel local ${ }^{2}$. En segundo lugar, esta institucionalización tuvo un importante impacto en la vida del paciente, al crear unas nuevas condiciones objetivas que cambiaron totalmente su experiencia de enfermar. Lo que antes se había desarrollado en un entorno familiar o en instituciones enfocadas más al cuidado del alma que al del cuerpo, ahora tenía lugar en una institución progresivamente medicalizada conforme avanzó el siglo, dependiente del Estado, con todo lo que ello implica, a lo que se debe añadir una práctica terapéutica que no conseguía ni curar su enfermedad, ni paliar sus síntomas ${ }^{3}$.

Para conocer exactamente esta nueva situación atenderemos a las prácticas concretas desarrolladas en los hospitales para incurables. Para ello seguiremos distintos caminos. En primer lugar, prestaremos atención a los reglamentos de los hospitales de incurables, buscando capturar el día a día de los enfermos en la institución, sus normas de funcionamiento, los procesos de inclusión, y demás elementos relevantes. En segundo lugar, estudiaremos los expedientes de los enfermos acogidos en el Hospital Nuestra Señora del Carmen a lo largo del año 1891, una de las series mejor conservadas y con mayor número de documentos, exactamente treinta y tres. De esta forma, trataremos de determinar quién era considerado «enfermo incurable»a finales del siglo XIX y qué criterios debía cumplir para formar parte de dicha categoría. El hecho de limitarnos a las instituciones de incurables, pese a saber de la existencia de otras en que también es posible encontrar

2. Zaragoza, Juan Manuel. La palabra más brutal: definiciones de enfermedad incurable en la medicina francesa del siglo XIX. Asclepio, 2012, en prensa. Este debate estaba unido al de una definición de qué era ser «pobre», intentando superar la antigua distinción entre «pobres verdaderos» y «pobres falsos», véase Franco Bilbao, Javier; Macías, Olga. Contextos y orígenes del hospital San Antonio Abad de San Sebastián. Bilbao: Museo Vasco de Historia de la Medicina y de la Ciencia; 2009, p. 40.

3. La historia de la enfermedad ha gozado de gran popularidad en los últimos años, especialmente desde los inicios de la década de 1990, tras el impacto que supuso la pandemia de SIDA de los 80 , véase Arrizabalaga, Jon. Historia de la enfermedad: nuevos enfoques y problemas. Presentación. Dynamis. 1991; 11: 17-26. Los libros relevantes son numerosos, y entre ellos encontramos propuestas generales sobre cómo abordar su estudio, como Rosenberg, Charles E.; Golden, Janet eds. Framing disease: Studies in cultural history. New Brunswick: Rutger University Press, 1997, hasta trabajos sobre enfermedades particulares, como Holland, Michael; Gill, Geoffrey; Burrel, Sean, eds. Cholera and conflict: 19th century cholera in Britain and its social consequences. Leeds: Medical Museum Publishing; 2009. El común denominador de estos escritos, pese a su diversidad, reside en la consideración de la enfermedad como una construcción social, no como entidades naturales y por tanto transhistóricas. Como bien dice Arrizabalaga en el texto citado, la recepción de Ludwik Fleck en la década de los ochenta del pasado siglo fue la causa principal de esta nueva aproximación a la enfermedad. Esa es, también, la perspectiva adoptada en el presente texto. 
enfermos crónicos o incurables, se debe a que la particular forma de ingreso en estos hospitales, mediante una petición, nos permite conocer cuáles eran los síntomas más señalados por parte de los enfermos y hacernos una idea de sus principales sufrimientos. Por otra parte, si bien el criterio de ingreso era restrictivo, sí es cierto que se explicita en la documentación cuándo un enfermo no es aceptado en el hospital por padecer una enfermedad incurable «con calentura», y cuándo no lo es porque su enfermedad no es incurable. De esta forma, en principio, el hospital de incurables será un sitio en el que todos sus residentes serán enfermos incurables, si bien no todo tipo de enfermedad incurable esté representada en la muestra. Éste es otro motivo por el cual restringimos la pesquisa sobre las enfermedades incurables al entorno institucional, cerrado y controlado, del Nuestra Señora del Carmen. La lectura de estos expedientes nos permitirá, por otra parte, conocer otros datos de interés sobre los enfermos que poblaban sus salas: su edad, su constitución física, sus capacidades, sus limitaciones, etc. En tercer y último lugar, buscaremos qué remedios eran aplicados a los enfermos y hasta qué punto eran adecuados para tratar las dolencias que sufrían.

Pero antes deberemos encuadrar, siquiera someramente, estos hospitales en su contexto institucional y político.

\section{La reforma de la beneficencia}

El Hospital para Hombres Incurables Nuestra Señora del Carmen fue fundado como tal en 1852, aunque su historia se remonta hasta el año 1592, en que la Congregación del Amor de Dios decidió fundar un asilo para niños desamparados. Sería sin embargo en 1852 cuando, a instancias del Gobernador Civil de la Villa de Madrid, los niños fueron trasladados a otra localización, convirtiéndose en hospital para incurables. Como tal, siguió funcionando hasta 1949, llegando a contar con doscientas noventa camas $^{4}$. La fecha de 1852 es importante, porque en ese preciso momento se produjo un cambio profundo en la estructura de la asistencia hospitalaria en España de manos del gobierno liberal ${ }^{5}$. La estructura de la beneficencia en

4. Álvarez-Sierra, José. Los hospitales de Madrid de ayer y de hoy. Madrid: Artes Gráficas Municipales; 1952, p. 61-62.

5. Maza Zorrilla, Elena. Pobreza y asistencia social en España: siglos XVI al XX. Valladolid: Universidad de Valladolid; 1987, p. 180-186. También en la década de 1850 se reclama desde la 
España se había caracterizado tradicionalmente por una fuerte presencia de instituciones religiosas que formaban lo que se daba en llamar beneficencia particular, mucho más extendida que la pública o del reino. Con la llegada al poder de los liberales, y más concretamente de los moderados, se inició una profunda reforma del sistema:

«He aquí el servicio de la beneficencia completamente secularizado, y hecho una de las primeras funciones de la Administración pública. He aquí las bases de la reforma antes apuntada, y que viene elaborándose lentamente, consultando las creencias y las costumbres de los pueblos, y estudiando y buscando remedio a sus nuevas necesidades ${ }^{6}$.

Este cambio tuvo su origen en un concepto de la beneficencia entendida como el conjunto de las instituciones públicas y privadas dedicadas al socorro de los pobres, basado en la virtud cristiana de la caridad, entendida como el sentimiento o virtud privada que estaba detrás de la beneficencia, servicio administrativo ${ }^{7}$ : «La Beneficencia pública es un deber social moral, fundado en la equidad y encerrado en los reducidos límites de la posibilidad de cumplirlo y de no lastimar los derechos perfectos de los asociados» 8 .

Basada en estos planteamientos, la reforma de 1845 clasificó los establecimientos benéficos en provinciales y municipales, clasificación que se amplió, en la Ley General de Beneficencia y Sanidad de 1849, para incluir aquellas instituciones que eran competencia directa de la administración general del Estado. De esta forma, la ley estableció tres grandes niveles de

prensa inglesa, en una campaña encabezada por Dickens y su periódico Household Words, la creación de un hospital para incurables, ante la ausencia de una institución que se hiciera cargo de estos enfermos. Hasta ese momento, los enfermos incurables pobres eran acogidos en las workhouses y en las Almhouses, o en algunas instituciones que tenían unas pocas plazas reservada para ellos. El Royal Hospital for Incurables se fundaría en 1854, tan solo dos años más tarde que el Nuestra Señora del Carmen, véase Cook, Gordon Charles. Victorian incurables: a history of the Royal Hospital for Neuro-Disability, Putney- Durham: Memoir Club; 2004, p. 1-2. Para la situación en Francia, son útiles Reix, Nicolas; Bois, Gérard. Les médecines face aux mourants. Paris: Connaissances et Savoirs; 2004, o el reciente trabajo: Szabo, Jason. Incurable and intolerable. Chronic disease and slow death in nineteenth-century France. New Brunswick: Rutgers University Press; 2010, así como Zaragoza, n. 2.

6. Hernández Iglesias, Fermín. La Beneficencia en España. Madrid: Establecimientos tipográficos de Manuel Minuesa; 1876, vol. 1, p. 86-87. Véase, también, Carasa Soto, n. 1, p. 54-55.

7. Hernández Iglesias, n. 6, p. 131. Esta definición fue ampliamente discutida y finalmente adoptada por los teóricos de la administración pública. Franco Bilbao; Macías, n. 2, p. 33 y ss.

8. Hernández Iglesias, n. 6, p. 134. 
competencia asistencial: el general, el provincial y el municipal ${ }^{9}$, cada nivel con sus competencias particulares. Los hospitales para aquellos enfermos con necesidades permanentes o especiales dependían, directamente, de la Administración General del Estado:

«Pero quiere la Ley que solo sean establecimientos generales los destinados exclusivamente a satisfacer necesidades permanentes o que reclaman atención especial. A esta clase pertenecen, dice, los establecimientos de locos, sordo-mudos, ciegos, impedidos y decrépitos» ${ }^{10}$.

Esta división se vio confirmada en el Real Reglamento del 14 de mayo de 1852, que daba un nuevo empuje a las reformas iniciadas con la ley de $1849^{11}$. Una de las principales aportaciones de esta reforma fue la creación de una Dirección General de Beneficencia, dependiente del Ministerio de Gobernación (posteriormente Interior) y que se haría cargo de «lo perteneciente a hospitales, hospicios, casas de refugio, de perseverancia y de maternidad, establecimientos de dementes y sus análogos, montes de piedad, limosnas y socorro públicos, e indemnizaciones por desgracia o calamidades» ${ }^{12}$. Dicha Dirección General pervivió, bajo distintos nombres, hasta la creación del Ministerio de Sanidad en 1977.

El Hospital Nuestra Señora del Carmen formaba parte, por tanto, de las dependencias gestionadas por la administración general, junto a otras destinadas a satisfacer «necesidades permanentes», como el Hospital para dementes Santa Isabel, sito en Leganés, el Hospital para Mujeres Incurables Jesús Nazareno, también en Madrid, o el Hospital del Rey, en Toledo, para decrépitos, hombres y mujeres ${ }^{13}$. La escasez de plazas en estas instituciones generaba una serie de problemas fáciles de imaginar: listas de espera

9. Hernández Iglesias, n. 6, p. 90 y 98. Barona Vilar, Carmen. Las políticas de la salud. La sanidad valenciana entre 1855 y 1936. Valencia: Universitat de València; 2006, p. 41.

10. Hernández Iglesias, n. 6, p. 134.

11. Hernández Iglesias, n. 6, p. 97.

12. Hernández Iglesias, Fermín. La Beneficencia en España. Madrid: Establecimientos Tipográficos de Manuel Minuesa; 1876, vol. 2, p. 741-742.

13. Hernández Iglesias, n. 6, p. 292. También Carasa Soto, n. 1, p. 46. La literatura sobre estos hospitales (con la notable excepción del Manicomio de Leganés) es escasa. La única obra que hemos localizado sobre los hospitales de incurables, dedicada al hospital para mujeres Jesús Nazareno, es la de Vidal Galache, Florentina. Ser viejo en Madrid. El Hospital de Incurables de Jesús Nazareno y otros centros de asistencia a los ancianos. Espacio, Tiempo y Forma. Historia Contemporánea. 1993; 6: 367-376. 
interminables, instituciones de caridad privada que ocupaban el espacio de la administración y, por último, el ingreso de muchos enfermos incurables o crónicos en hospitales para agudos, con la sobrecarga asistencial y financiera que esto supone. En un informe estadístico sobre el Hospital General de Madrid en el año 1853 se consigna explícitamente como causa de tal sobrecarga, por un lado, el escaso número de plazas con que los hospitales para incurables, tanto para hombres como para mujeres, cuentan, y por otro, la misma naturaleza de las enfermedades allí admitidas: sólo aquellas que no «ofrece[n] calentura» ${ }^{14}$.

\section{El Hospital de Incurables Nuestra Señora del Carmen}

\subsection{Reglamentos para la gestión de los Hospitales de Incurables}

El reglamento para la gestión de los hospitales de incurables dependientes de la Subdirección de Beneficencia vigente en el año 1891 fue publicado en la Gaceta de Madrid el 5 de febrero de 1885, y englobaba por primera vez a los tres hospitales de este tipo ${ }^{15}$, ya que, hasta ese momento, cada uno de ellos había gozado de sus propios estatutos. De forma general, en el reglamento de 1885 se especificaba el tipo de enfermos que podían ingresar en estos hospitales: $1^{\circ}$. Enfermos crónicos infebriles que requieran cuidados continuos; $2^{\circ}$. Impedidos por enfermedad crónica o vicio orgánico congénito o adquirido ${ }^{16}$. En un artículo posterior del reglamento, se marcan otro tipo de condiciones para el ingreso, delineando, de esta forma, aun más el perfil de nuestros enfermos. Estas condiciones son: 1. Que los ancianos sean mayores de 65 años y no dispongan de medio de subsistencia, ni familia

14. En dicho informe estadístico se llega a afirmar que dos terceras partes de los enfermos tratados en el hospital eran crónicos o incurables, véase de Llanos, José María. Apuntes estadísticos sobre el Hospital General de Madrid, recogidos por D. J. M. de Llanos. Boletín de Medicina, Cirugía y Farmacia, Segunda Época. 1853; III (114), 74-79, situación que no mejoraría con el paso del tiempo. En un artículo publicado el 16 de Agosto de 1918 en la revista Nuevo Mundo sobre el Hospital Provincial de Madrid (antiguo Hospital General), el director administrativo del mismo afirmaba que los enfermos incurables y ancianos ocupaban el sesenta por ciento de las plazas del hospital: En el mundo del dolor. Una visita al Hospital Central. Nuevo Mundo. 16 Oct 1916.

15. Reglamento para el orden interior de los Hospitales de Incurables. Gaceta de Madrid. 5 Feb 1885; 33: 361-363.

16. Reglamento, n. 15, p. 361. 
que pueda sostenerlos; 2. Que los impedidos sean mayores de 50 años y cumplan idénticos requisitos de «pobreza y abandono» ${ }^{17}$. En el artículo 18 del mismo capítulo, se especifican todavía más condiciones de acceso:

«No podrán ser admitidos en estos hospitales: $1 .^{\circ}$ Los que tengan hijos solteros mayores de edad o menores que no acrediten debidamente que los tienen abandonados sin causa justificada; $2 .^{\circ}$ Los que padezcan enfermedades contagiosas; $3 .^{\circ}$ Los enajenados, los idiotas, imbéciles, epilépticos y tuberculosos; los que padezcan úlceras con supuraciones incoescibles [sic], los cánceres externos y aquellos que para su curación necesiten una operación quirúrgica» ${ }^{18}$.

Nos encontramos, por tanto, con una población de edad avanzada, impedida por sus enfermedades a valerse por sí misma y sin ningún apoyo familiar, ya sea económico o moral. Son, en el sentido más literal de la palabra, ancianos solitarios y sin esperanzas, que han quedado fuera de cualquier tipo de relación social (laboral, familiar) y cuya única posibilidad era ingresar en alguna de estas instituciones.

Procedamos a situar a nuestros enfermos en su sitio. El Hospital de Nuestra Señora del Carmen ocupaba un edificio relativamente grande, situado en la calle de Atocha, esquina con Costanilla de los Desamparados. Construcción renacentista, de finales del reinado de Felipe II, su fachada de 190 pies castellanos (52,44 metros) se abría (y se abre) a la calle de Atocha, contando con una puerta frontal, descentrada a la izquierda del edificio, y amplios ventanales, enrejados en la planta baja y con pequeños balcones en la superior. Adosada al edificio, se encuentra la Iglesia del Santísimo Cristo de la Fe, utilizada como capilla para los internos. En la trasera, un patio de grandes dimensiones, con jardín y arbolado, empleado para solaz de los enfermos ${ }^{19}$. En su interior, cocinas, almacenes, oficinas, una despensa, un comedor para los internos y otro para los trabajadores, estancias para el recreo, habitaciones para las Hijas de la Caridad que cuidaban de los acogidos y del funcionamiento diario del hospital, habitaciones para el administrador, el comisario interventor, dependientes con derecho a alojamiento (practicantes, enfermeros, mozos), la enfermería y, por fin, las habitaciones de los

17. Reglamento, n. 15, p. 362.

18. Reglamento, n. 15, p. 362.

19. Domingo Mambrilla, Clemente. La Beneficencia Pública. Revista de España. Tomo CXLVIII. Madrid: Est. Tip. Ricardo Fe; 1894, p. 432. 
enfermos $^{20}$. El número de enfermos variaba. Mientras que los reglamentos marcaban un máximo de 250 enfermos $^{21}$, un documento del año 1886 cifra, a finales de noviembre de dicho año, el total de enfermos en 252. Durante el mes de diciembre del mismo año, el total había descendido hasta $237^{22}$. No podemos menos que preguntarnos cómo podía caber tanta estancia y tanta gente en su interior. El edificio debía estar abarrotado. Ayudaba a ello la escasa distancia que, por ley, debía existir entre una y otra cama del enfermo: 75 centímetros. El espacio necesario para situar una mesilla en la que depositar recipientes y vasijas ${ }^{23}$. La intimidad de los enfermos, intuimos, era un concepto inexistente. Y pese a todo, no debía ser un lugar tan terrible para vivir, a tenor de algunos testimonios:

«Llama la atención el Establecimiento por la limpieza, aseo y orden que en él reina: las distintas salas, bien ventiladas, con un determinado número de asilados, en cada una, y teniendo cada enfermo ó decrépito un buen lecho, ropas de cama limpias, y con el abrigo necesario, y todo aquel mobiliario indispensable, atendida la edad y achaques, recorriendo aquellas salas, se vé [sic] con frecuencia á algunos en cómodos sillones, á otros en canapés apropósito, y todos perfectamente asistidos y cuidados por el personal que, con celo y cariño, les prodiga toda clase de atenciones haciéndoles sobrellevar mejor los dias de la vejez» 24 .

El panorama descrito por Domingo Mambrilla es idílico. Podemos casi imaginar a los ancianos recostados en sus canapés, disfrutando del calor del sol madrileño que entra por los amplios ventanales del edificio, mientras una Hija de la Caridad atiende hasta la última de sus necesidades, en una estancia limpia, perfumada... sin embargo, su juicio contrasta profundamente con el de otro testigo de la época, tal vez menos autocomplaciente:

«No siendo ahora nuestro objeto en este capítulo de describir los hospitales como establecimientos de beneficencia, sino bajo el punto de vista de la policía sanitaria, nos limitamos á hacer constar que estos dos últimos [el Jesús Nazareno y el Nuestra Señora del Carmen] carecen completamente de las condiciones higiénicas exigidas para un establecimiento de esta índole

20. Reglamento, n. 15, p. 362.

21. Reglamento, n. 15, p. 362.

22. Archivo Histórico Nacional (en adelante AHN), legajo 214/44.

23. Reglamento, n. 15, p. 362.

24. Domingo Mambrilla, n. 19, p. 431-432. 
[...] El de Nuestra Señora del Carmen, aunque se halla provisto de un patio grande con jardín y arbolado, la aglomeración de un número crecido de asilados enfermos en un recinto relativamente pequeño, constituye un foco de insalubridad dentro de una calle tan populosa como es la de Atocha» ${ }^{25}$.

Poco tiene que ver el «foco de insalubridad» de Hauser con esa especie de balneario descrito por Domingo Mambrilla. ¿Qué imagen debemos guardar en nuestra retina? Posiblemente la verdad se encuentre en un punto medio. No nos resultará difícil, dado el número de personas recluidas en un espacio «relativamente pequeño», desterrar de nuestra mente esa imagen de enfermos en canapés tomando el sol. Pero tampoco debemos pensar que los enfermos estaban abandonados a sí mismos, lejos de toda atención sanitaria e higiénica. No obstante, las críticas al trato recibido por los enfermos eran bastante comunes. Concepción Arenal, en uno de los numerosos artículos que escribió contra el proyectado traslado de los establecimientos de beneficencia desde el centro de Madrid al edificio que se debía construir en la Dehesa de Amaniel, argumentaba lo siguiente:

«Además, estas persona que van á consolar á los pobres, son el público para los empleados, y aunque por desgracia no sea muy eficaz su presencia para correjir [sic] abusos, aunque no sea muy fuerte el freno de su crítica para contener desmanes, quien conozca el corazón humano y los establecimientos de Beneficencia, ¿puede dudar de que si las cosas no van hoy bien, irán peor cuando estén aislados, cuando nadie vea ni sepa lo que en ellos pasa?» ${ }^{26}$.

Lo cierto es que el régimen de encierro facilitaba ese abuso. A los enfermos se les alimentaba, se les proporcionaban ropas limpias y adecuadas, tanto para diario como para los días festivos, un pañuelo que se le cambiaba todas las semanas... y sin embargo, los internos debían, por fuerza, echar de menos productos del exterior. Las comidas eran monótonas, y muchas veces se repetirían las sopas con pan en el desayuno, la comida y la cena ${ }^{27}$. La bebida, el tabaco, los juegos de naipes... todo eso estaba prohibido y

25. Hauser, Philiph. Madrid bajo el punto de vista médico-social. Madrid: Editora Nacional; 1979 [1. ${ }^{\text {a ed. }}$ 1903].

26. Arenal, Concepción. ¡Pobres pobres! La Iberia. 28 Nov 1868: 1.

27. Reglamento, n. 15, p. 362. Fuera del hospital la dieta del español pobre no era tampoco variada. Para un periodo posterior pero probablemente similar, consultar Del Cura, Isabel; Huertas, Rafael. Alimentación y enfermedad en tiempos de hambre, 1937-1947. Madrid: CSIC; 2007. 
castigado $^{28}$. No nos puede extrañar que el tráfico desde el exterior de productos más o menos inocentes como unos pastelillos, algo de fiambre o una botella de licor fuera materia común. Hasta qué punto se realizaba con la complicidad comprada del personal del hospital, especialmente el portero, es algo que sólo podemos conjeturar ${ }^{29}$. Las posibilidades para el abuso en un sitio como el Hospital de Nuestra Señora del Carmen eran numerosas. Por otra parte, la prohibición explícita de los castigos físicos en el reglamento nos empuja a pensar que éstos fueron frecuentes ${ }^{30}$.

Nos gustaría tratar un punto más, antes de pasar al análisis de las peticiones de ingreso. Se trata de los permisos para estancias temporales y el régimen de visitas y salidas de los enfermos. En lo que respecta a las primeras, el Reglamento del centro las limita de forma expresa a aquellas que estuvieran motivadas por causas de salud, con la recomendación del facultativo, y su única finalidad debía ser que el enfermo acudiese a recibir baños de aguas minero-medicinales en un balneario ${ }^{31}$. Estas estancias, que no podían ser superiores a tres meses, eran improrrogables. Hay un dato, sin embargo, que nos parece importante señalar: muchos de los que salían, nunca volvían al hospital. Y no siempre era la muerte, ni una mejoría de su enfermedad, la causa de su ausencia: «Cumplido el término de la licencia sin que se presente el albergado, será dado de baja en el establecimiento y cubierta su plaza» ${ }^{32}$.

Otro punto de interés son las visitas y salidas de los internos. Nada nos dicen los estatutos de las visitas, pero sí de las salidas. Según los estatutos, la junta debía permitir a los enfermos la salida en los días festivos, en horas determinadas. Esta salida estaría siempre controlada por encargados designados por el administrador y, en el caso de las mujeres, por Hijas de la Caridad $^{33}$. En cuanto a las visitas, poco o nada podemos extraer de los

28. Ver, por ejemplo, el legajo 2022/37 del AHN, en el que se describen las medidas tomadas contra unos enfermos que fueron sorprendidos mientras jugaban a las cartas. La Junta de Patronos recomendaba la expulsión de dichos internos, al tratarse de reincidentes.

29. Entre las funciones del portero estaba la de evitar que se introdujeran productos desde el exterior, el poder que tal tarea le otorgaba no puede ser despreciado. Reglamento, n. 15, p. 363.

30. Reglamento, n. 15, p. 363.

31. Reglamento, n. 15, p. 363, Capítulo XXII, Art. 89. La documentación de archivo confirma este punto, véase AHN, legajo 1402/28-42.

32. Reglamento, n. 15, p. 363.

33. Reglamento, n. 15, p. 363. 
reglamentos. La única referencia la encontramos en una carta escrita al periódico La Iberia, en el año 1868:

«Un individuo del hospital de hombres incurables de Nuestra Señora del Carmen nos dirije una carta [...] En la espresada [sic] carta se consigna el deseo de que se permita la entrada todos los domingos por la mañana y tarde en el establecimiento, pues es muy triste para aquellos desgraciados tener que pasar quince dias sin ver á su familia, y por consiguiente, sin recibir los consuelos que tanto necesitan ${ }^{34}$.

Podemos, por tanto, completar una imagen de nuestros enfermos aislados del mundo exterior, protegidos de él. Las salidas están controladas por supervisores del hospital. Las visitas son escasas, cada quince días, no pueden introducir nada desde el exterior y, además, debían ser autorizadas expresamente por el administrador del hospital o el visitador de beneficencia $^{35}$. Por otra parte, podemos constatar que los enfermos no aceptaban pasivamente esta situación. La carta a La Iberia es una evidente prueba de resistencia, si bien débil, a la autoridad del hospital. Aún más las fugas que el artículo 91 del reglamento parece suponer. El panorama que hemos deducido es bastante diferente al expuesto por Domingo Mambrilla. Si se quiere, algo más siniestro. Nos encontramos con una historia de pequeñas resistencias ante una situación que no es, ni mucho menos, idílica.

\subsection{Peticiones de ingreso}

El método de ingreso era complicado. Según indican los estatutos, el enfermo elevaba una solicitud de ingreso al Subdirector General de Beneficencia ${ }^{36}$. Esta solicitud tenía la forma de un «expone y solicita». En la primera parte, el solicitante exponía cuál era su situación, la enfermedad incurable que sufría (la mayoría de las veces lo que se relataban eran los síntomas, aunque encontramos algunas solicitudes en las que se nombra específicamente la enfermedad y se califica como incurable, acompañadas, normalmente, de un informe médico previo), y que a causa de dicha enfermedad se encontraba incapacitado para trabajar. En la segunda parte del documento, el enfermo

\footnotetext{
34. Cartas al director. La Iberia, 6 Dic 1868: 2.

35. Reglamento, n. 15, p. 363.

36. Reglamento, n. 15, p. 362.
} 
solicita su ingreso en el hospital en una de las categorías señaladas en el reglamento ${ }^{37}$. Esta petición solía ir apoyada por otros informes que daban fe de la veracidad de lo expuesto, ya sea por parte del alcalde de barrio, del cura párroco, de un médico, etc. Adjunta, la partida de bautismo y, a veces, un certificado de buena conducta, una carta de presentación, etc. Algunos adjuntaban incluso el documento que les acreditaba como reservistas del ejército ${ }^{38}$. Toda esta documentación era enviada desde la Subdirección General de Beneficencia a la Junta de Patronos del Hospital, para que se incoara el expediente. Se suceden, a partir de este momento, las averiguaciones. En primer lugar toma la palabra el facultativo, que debe diagnosticar la enfermedad del solicitante y ver si se ajusta al reglamento, en cuyo caso recomienda su admisión. Este informe médico se complementa con otro en que se da fe de las buenas costumbres del mismo, así como de la veracidad de su estado de pobreza ${ }^{39}$. Ambos son entregados de nuevo a la Junta que, tras su estudio, procede a admitir o rechazar la petición. En caso de aceptarse, se le adjudica un número de turno y se eleva todo el expediente, nuevamente, a la Subdirección General, que da el visto bueno y lo comunica a la Junta de Patronos y al interesado. El proceso solía extenderse durante unos cuatro meses. Pero su finalización no significaba el ingreso automático del enfermo. Éste debía esperar a que llegase su turno, a que hubiera plaza. No es de extrañar, por tanto, que el proceso se dilatase en el tiempo. En un estudio realizado sobre las 33 peticiones de ingreso contenidas en el legajo 1984 del Archivo Histórico Nacional (AHN) correspondientes al año 1891, hemos podido comprobar que el $45 \%$ de los solicitantes admitidos murieron antes de obtener una plaza. Tres de los solicitantes debieron solicitar plaza como medio pensionistas (pagando una peseta diaria) tras haber obtenido plaza como pobre para poder ingresar en el hospital, y la media de tiempo que se tardaba entre la petición de ingreso y el ingreso definitivo como pobre era de 586 días, más de un año y medio ${ }^{40}$.

\footnotetext{
37. Pobre, medio pensionista o pensionista, Reglamento, n. 15, p. 362.

38. Según el Reglamento, n. 15, p. 362, la solicitud debía ir acompañada de la partida de bautismo, certificación de pobreza expedida por el alcalde y un informe facultativo acreditando el estado del enfermo.

39. Sobre el informe médico, Reglamento, n. 15, p. 363; del informe sobre la moralidad del individuo, era una obligación de la Junta de Patronos, máxima responsable del funcionamiento del Hospital, tal y como se indica en Reglamento, n. 15, p. 361.

40. La media de tiempo para ingresar como medio pensionista era tan sólo de 47 días. No se ha incluido en este cálculo aquellos enfermos que, admitidos como pobres, debieron pedir su
} 
Respecto a la edad de los internos, el dato que se desprende de las peticiones del año 1891 es contundente: la media de edad de los peticionarios era de 67 años. El más viejo tenía 84 . El más joven, 52. Ninguno de los dos llegó a ingresar. Murieron en la espera. Esta media de edad explica también muchas de las enfermedades que alegaban, alegaciones que solían venir acompañadas, en la mayoría de los casos, por una expresión que, por repetida, ya adquiere naturaleza de fórmula: «y otros achaques propios de la avanzada edad».

\subsection{Enfermedades y remedios}

En el presente punto vamos a tratar sobre síntomas, enfermedades, remedios y cuidados. Tal vez sea esto lo primero que nos viene a la mente a la hora de hablar de la enfermedad: aquello que los médicos dicen y hacen. El situarlo en este lugar del texto no es una decisión casual. Hay dos razones que nos empujan a ello: la primera, hacer relevante que, por muy importante que pueda ser, se trata solamente de una faceta más de la experiencia de enfermar; la segunda, el papel mismo que la medicina tiene en el Hospital de Nuestra Señora del Carmen:

«Ambas redes asistenciales - la pública y la privada - siguieron caminos muy diferentes a lo largo del siglo XIX. Así, los manicomios públicos españoles tuvieron serias dificultades para convertirse en espacios medicalizados: la ausencia de directores médicos en los mismos se ha argumentado repetidamente como una de las principales causas del deterioro de las instituciones. Instituciones, regidas por órdenes religiosas o, a lo sumo, por patronatos. En las que los médicos permanecieron relegados a una simple función de "asesores" o de "jefes facultativos"»"

El Hospital del Carmen no era una excepción. La labor del médico, tal y como se describe en el reglamento, sería completamente técnica: visitar a los enfermos, practicar operaciones, recetar, realizar un estado semestral

ingreso como medio pensionistas, pues entendemos que resulta más interesante seguir el proceso de ingresos de éstos desde el principio, y cuantificar el tiempo transcurrido desde su primera petición hasta el ingreso mismo. Obviamente, la media entre estos enfermos es menor, quedando tan sólo en 349 días.

41. Huertas, Rafael. Los laboratorios de la norma. Medicina y regulación social en el Estado liberal. Barcelona: Octaedro; 2008, p. 55. 
para el visitador del ministerio, reconocer al paciente en el ingreso, etc. ${ }^{42}$ Incluso una faceta tan importante para la medicina de la época como era la de marcar la dieta del enfermo debía ser aprobada por la Junta de Patronos ${ }^{43}$. La relación del paciente con la medicina y el médico son, por tanto, sólo una parte más de la experiencia de enfermar, como decíamos, pero en un hospital de beneficencia del siglo XIX esto es más evidente que en ningún otro sitio ${ }^{44}$.

Hay dos tipos de documentos que nos interesan en la identificación de las enfermedades que sufrían los aspirantes a internos. Por un lado, tenemos la petición de ingreso. En ella, como ya hemos dicho, el enfermo, por mano propia o la mayor de las veces ajena, relataba cuáles eran los males que sufría, esos síntomas que constituían su enfermedad. Por otra parte, el informe médico del facultativo del hospital, en el que los síntomas descritos toman forma bajo la etiqueta de una entidad nosológica: una enfermedad. Ambos discursos se complementan y nos permiten acceder a dos realidades distintas: por un lado, la experiencia concreta del enfermo, que resalta aquellos síntomas que tienen un especial significado en su vida, los síntomas que realmente le hacen sufrir una enfermedad. Por otro lado, el entramado científico de las enfermedades incurables, un listado de aquellas dolencias que, realmente, eran incurables. Al analizar la lista resultante de escuchar ambas voces se descubre una curiosa realidad: aquello que importa al enfermo no es necesariamente lo que importa al médico.

Empecemos por los enfermos. Contamos con 32 peticiones completas de los 33 peticionarios que vienen recogidos en el legajo 1984. En estas 32 peticiones se recogen un total de 41 causas por las que se solicita dicho ingreso. De esas 41, tan sólo once hacen referencia a una enfermedad concreta (27\%), tres de ellas respaldadas por un informe médico externo: mielitis (1), infarto de próstata (1), hemiplejia (1), paraplejia (1), ataxia

\footnotetext{
42. Reglamento, n. 15, p. 362-363.

43. Reglamento, n. 15, p. 362. No se debe restringir estos datos a los Hospitales de Incurables, véase Franco Bilbao; Macías, n. 2, p. 60 y 62, donde se describe el proceso de admisión en el Hospital y Casa de Beneficencia de Sabadell hacia 1887.

44. Tanto la historia de la medicina desde la perspectiva del paciente como la antropología médica han señalado ampliamente este punto. Véase, para la primera, el texto clásico de Porter, Roy. The patient's view: Doing medical history from below. Theory and Society. 1985; 14 (2); p. 175-198, así como Condrau, Florence. The patient's view meets the medical gaze. Social History of Medicine. 2007; 20 (3); 525-540. Sobre antropología de la enfermedad, y los distintos aspectos de la experiencia de enfermar, consúltese el clásico de Kleinman, Arthur. Suffering, healing and the human condition. New York: Basic Books; 1988.
} 
locomotriz (1), catarro pulmonar crónico (5), y asma (1). El resto de las peticiones describen síntomas, percepciones o denominaciones populares: parálisis del lado derecho, inútil, quebrado, imposibilitado de la vista, etc. Entre estos síntomas hay uno que, sin lugar a dudas, destaca poderosamente: el dolor. Catorce de los cuarenta y un enfermos declara que «sufre dolor», sea de origen reumático (el más común) o «nervioso». Es decir, el 34\% de los principales síntomas declarados por los solicitantes, aquellos síntomas que, en su opinión, deben abrirles las puertas del Hospital de Incurables, tienen que ver con el dolor. El siguiente síntoma o enfermedad en número de apariciones es el catarro crónico: significa tan sólo el 12\% del total de los síntomas. No cabe duda, por tanto, de cuál era la principal preocupación de los enfermos.

El listado proporcionado por los médicos nos muestra otra realidad, cuya principal diferencia estriba en la desaparición del dolor como elemento denominador. El dolor, para el médico, no deja de ser un síntoma más que forma parte del grupo que conforma la definición de una enfermedad ${ }^{45}$. El listado es extenso, y comprende 24 enfermedades distintas que aparecen un total de 47 veces. En la Tabla 1 se expone, ordenadas según el número de apariciones, la lista completa de enfermedades diagnosticadas.

Como vemos, la enfermedad que aparece diagnosticada con más frecuencia es el reumatismo, en correspondencia con los frecuentes «dolores reumáticos» manifestados por los peticionarios. Hemos resumido bajo esta etiqueta los tres tipos de reumatismo descritos en los informes médicos, a saber: articular (7), fibroso (1) y nervioso (1). A continuación encontramos los catarros pulmonares crónicos (7), hernias (6) y hemiplejias (4), como enfermedades más comunes. El resto de enfermedades, con una sola aparición, son bastante variadas. Al igual que la lista anterior nos indicaba qué tipo de síntomas eran los más importantes para los pacientes, la contenida en la tabla número 1 nos aporta un listado con 24 enfermedades que, en el año 1891, eran consideradas incurables.

\subsection{Recetas y cataplasmas}

La medicación que se administraba en ambos hospitales de incurables de Madrid, tanto para hombres como para mujeres, era servida por la botica

45. King, Lester. What is disease? Philosophy of Science, 1954; 21; 193-203 (197). 
Tabla 1. Relación de enfermedades diagnosticadas por los facultativos del Hospital de Incurables Nuestra Señora del Carmen

\begin{tabular}{|c|c|c|}
\hline & Enfermedad & Frecuencia \\
\hline 1 & Reumatismo & 9 \\
\hline 2 & Catarro pulmonar & 7 \\
\hline 3 & Hernias & 6 \\
\hline 4 & Hemiplejia & 4 \\
\hline 5 & Debilidad senil & 2 \\
\hline 6 & Hipoglobulia & 1 \\
\hline 7 & Infarto prostático senil & 1 \\
\hline 8 & Catarro bronquial & 1 \\
\hline 9 & Estenosis aórtica & 1 \\
\hline 10 & Bronquiectasia & 1 \\
\hline 11 & Semiparálisis de los extensores de ambas piernas & 1 \\
\hline 12 & Parálisis del cuello de la vejiga & 1 \\
\hline 13 & Parálisis incompleta del lado izquierdo & 1 \\
\hline 14 & Nefritis albuminosa (mal de Brhaigt $[$ sic $]$ & 1 \\
\hline 15 & Bridas febrosas en los flexores de los dedos & 1 \\
\hline 16 & Esclerosis & 1 \\
\hline 17 & Paraplejia & 1 \\
\hline 18 & Cataratas & 1 \\
\hline 19 & Asma & 1 \\
\hline 20 & Periencefalitis difusa & 1 \\
\hline 21 & Ataxia locomotriz & 1 \\
\hline 22 & Reblandecimiento cerebral senil & 1 \\
\hline 23 & Mielitis crónica & 1 \\
\hline 24 & Entero-colitis crónica & 1 \\
\hline
\end{tabular}

Fuente: Elaboración propia a partir de las peticiones de ingreso recogidas en AHN, legajo 1984.

del Hospital de la Princesa, que funcionaba a modo de farmacia central de ambos centros desde el año $1861^{46}$. Se enviaba, por tanto, de forma diaria una petición a dicha botica para el suministro de los medicamentos necesarios, firmada por el facultativo del hospital. Los medicamentos eran recibidos y almacenados en el botiquín, donde se encontraban a disposición de los 
facultativos. Por desgracia, la documentación no abarca todo el periodo. El grueso de la información se refiere a los años 1871 y 1872, concentrado en dos legajos del AHN (el 2094/3 y el 2094/8), y es extensísima, puesto que encontramos cada uno de los pedidos realizados a lo largo de casi un año. Unos trescientos listados de preparaciones que nos permiten hacernos una idea cabal del tipo de tratamiento que se practicaba en el hospital. En el presente texto, el análisis se reducirá a la documentación encontrada en el legajo 2094/3, que comprende los meses de septiembre, octubre, noviembre y diciembre de $1871^{47}$.

El estudio de esta parte de la documentación ofrece como resultado, en primer lugar, la aparición de un elevado número de opiáceos (clorhidrato de morfina, extracto acuoso tebaico, polvos de Dover). El principal uso de los opiáceos es, parece evidente, como analgésico, si bien también solían ser empleados como antitusivos y antidiarreicos, así como astringentes o en el tratamiento de disneas. Otros analgésicos, como el acónito ${ }^{48}$, también eran empleados regularmente. Junto a los analgésicos encontramos otros medicamentos que podemos dividir en dos grandes grupos: 1) indicados para el tratamiento de afecciones gastrointestinales: jarabe de altea, bicarbonato, nuez vómica; 2) indicados para el tratamiento de afecciones cutáneas o para preparar cataplasmas: harina de linaza, alumbre crudo.

Era necesario, pues así se reglamentó, que sólo se pudieran pedir las cantidades que se iban a emplear en las 24 horas siguientes, de forma que no se almacenase en los botiquines del hospital más medicación que la necesaria y, por supuesto, aquella de uso común (por ejemplo, esparadrapo) ${ }^{49}$.

47. Como se ha indicado hasta la saciedad, la continuidad de prácticas terapéuticas hipocráticas durante el siglo XIX contrasta con el abandono de esta corriente teórica. Así la costumbre del sangrado, como se verá, sigue vigente, así como el énfasis en la dieta y el ejercicio, prácticas de raigambre hipocrática que encuentran su cobertura teórica en la nueva práctica de la higiene: Bynum, William F. The rise of science in medicine, 1850-1913. In: Bynum, William F. et al. coords. The western medical tradition, 1800 to 2000. Cambridge: Cambridge University Press; 2006, p. 111-246. Un estudio ajustado de la historia del medicamento en los siglos XIX y XX en Bonah, Christian; Rasmussen, Anne. Histoire et médicament aux XIXe et XXe siècles. Paris: Éditions Glyphe, 2005. Una visión más amplia en Weatherall, Miles. In search of a cure. A history of pharmaceutical discovery. Oxford: Oxford University Press; 1990.

48. El acónito, del que suele emplearse la raíz, cuenta entre sus principios activos con la acotinina, en cantidades de hasta un 30\%, que le confiere propiedades analgésicas. Es empleado igualmente como antipirético, antitusivo y descongestionante. Como se ve, está especialmente indicado para afecciones del aparato respiratorio. Arteche, Alejandro. Fitoterapia, Vademecum de prescripción. Barcelona: Masson S.A.; 1998.

49. AHN, legajo 2141/10. 
Esto, que ofrece la ventaja de acercarnos al día a día de médicos y enfermos, implica también que las conclusiones obtenidas de la muestra no pueden ser definitivas. La distorsión de los datos se hace evidente en el empleo de clorhidrato de morfina. Este medicamento aparece desde mediados de octubre hasta el final de la muestra, en dosis de $10 \mathrm{dg}$. divididas en cuatro píldoras, muy probablemente para el tratamiento de un único enfermo durante un periodo de tiempo prolongado. Implica, por tanto, un peso desproporcionado en la muestra que, muy posiblemente, se corregirá con un estudio completo de la documentación que se antoja necesario. Por otra parte, no deja de llamar la atención el número relativamente escaso de medicamento solicitado si tenemos en cuenta el número total de internos, lo cual nos empuja a pensar que sólo algunos de ellos eran tratados farmacológicamente. Sí podemos, sin embargo, extraer una serie de consecuencias provisionales. La primera, que el principal síntoma tratado era el dolor producido por las enfermedades incurables de los internos. Apunta a ello el elevado número de opiáceos de diverso tipo y de otros analgésicos recetados. A continuación, las enfermedades respiratorias y gastrointestinales y las afecciones cutáneas. No entraremos de momento a analizar el éxito de tales tratamientos. Por otra parte, esta imagen extraída de la terapéutica aplicada se corresponde bastante bien con el cuadro de enfermedades y síntomas relacionados en el apartado anterior, si excluimos las afecciones cutáneas, que no se mencionan.

Nos centraremos ahora en las historias clínicas del Hospital Clínico de Madrid. Hemos utilizado para ello un libro publicado en 1882 por $E l$ Alumno Médico que recoge las historias clínicas recogidas por los alumnos del curso 1881-1882 durante sus prácticas de medicina clínica y de cirugía $^{50}$. Entre las muchas historias hemos escogido un total de 17. Tienen en común el tratarse de enfermedades crónicas (o con una alta probabilidad de convertirse en tales) e incurables. En estas 17 historias detectamos 11 enfermedades distintas, que detallamos en la Tabla 2:

50. Curso Académico de 1881-1882. Historias clínicas de los enfermos acogidos durante el citado curso en el Hospital Clínico de la Facultad de Medicina de Madrid recogidas y publicadas por la Redacción del «El Alumno Médico». Madrid: Administración; 1882. 
Tabla 2. Relación de enfermedades diagnosticadas por los facultativos del Hospital Clínico de Madrid (17 casos)

\begin{tabular}{|c|c|c|}
\hline & Enfermedad & Frecuencia \\
\hline 1 & Reumatismo & 4 \\
\hline 2 & Mielitis crónica & 3 \\
\hline 3 & Bronquitis crónica & 2 \\
\hline 4 & Cistitis crónica & 1 \\
\hline 5 & Cáncer de estómago & 1 \\
\hline 6 & Prostatitis crónica & 1 \\
\hline 7 & Meningitis tuberculosa & 1 \\
\hline 8 & Reumatismo muscular & 1 \\
\hline 9 & Catarro pulmonar & 1 \\
\hline 10 & Catarro gástrico crónico & 1 \\
\hline 11 & Atrofica muscular & 1 \\
\hline
\end{tabular}

Fuente: Elaboración propia a partir de 17 casos recogidos en el libro del Curso Académico 1881-82, n. 50.

Si lo comparamos con la Tabla 1, veremos que, nuevamente, el reumatismo se encuentra en lo más alto de la lista, con cuatro apariciones (un 24\% del total). El catarro pulmonar, sin embargo, que en el primero estaba en segundo lugar con 7 apariciones (el 15\% del total), aquí solo cuenta con 1 (6\%), si bien se ve acompañado por otros trastornos del sistema respiratorio, como la bronquitis crónica (12\%). Empecemos por estos cuatro casos, a los que sumaremos el de reumatismo muscular, que, pese a diagnosticarse agudo, se cree que derivará en crónico ${ }^{51}$. De los cuatro enfermos, dos son hombres y las otras dos mujeres. De los hombres, uno cuenta 65 años y el otro 19. De las mujeres, una 25 y la otra 23. En los cuatro casos, el primer síntoma referido son los dolores en las articulaciones, excepto en el caso de la mujer de 25 años, que menciona la pérdida de la menstruación en primer lugar ${ }^{52}$. Para describir dichos dolores se emplean adjetivos como «grandes» ${ }^{53} \mathrm{O}$ «intensos» ${ }^{54}$ y se indica el aumento de los mismos al moverse, hasta el punto de dificultar la locomoción. En los cuatro casos, los dolores, 
que comenzaron en las articulaciones de la rodilla, se desplazaron hasta las piernas y los tobillos y, en uno de ellos, hasta la cadera. Esto en lo relativo a los síntomas que presentaban los enfermos al entrar en el Clínico. Uno de ellos, el hombre de 65 años, desarrolló «edemas en las piernas» ${ }^{55}$, posiblemente un eritema nodoso. En cuanto al tratamiento, a las dos mujeres se les recomendó salicilato de sosa para combatir el dolor reumático (analgésico y antiinflamatorio) y el empleo de estufas secas en las articulaciones, para aportar calor ${ }^{56}$. En lo que respecta a los hombres, el tratamiento varió, dependiendo de sus casos particulares y de sus condiciones. El joven de 19 años, diagnosticado de artritis reumática, recibió baños con un cocimiento de manos de carnero en el pie derecho y una cataplasma emoliente (antiinflamatoria) tres veces al día. El 19 de febrero se le recomendaron unturas de pomada de belladona (analgésico local) y ungüento mercurial. El 11 de marzo, tras casi tres meses ingresado, se le recetan sanguijuelas aplicadas a los maléolos y unas embrocaciones de tintura de yodo por la mañana y por la tarde ${ }^{57}$. En el caso del anciano de 65 años, la medicación se redujo a una disolución de 2 gramos de yoduro potásico en 90 gramos de agua - posiblemente para tratar los nódulos-, fricciones con bálsamo al hipocondrio derecho, algún laxante y agua de Loeches ${ }^{58}$.

Podemos conjeturar, a la vista de las prescripciones, que la efectividad del tratamiento recibido sería más bien escasa, con el consiguiente impacto en las condiciones de vida del enfermo. Por otra parte, son habituales los tratamientos que contribuyen a incrementar la incomodidad del enfermo, así como su dolor. Es común la aplicación de cantáridas o papeles epispásticos, que producen grandes y dolorosas ampollas ${ }^{59}$; de botones de fuego ${ }^{60}$ para tratar la mielitis crónica; y nuevamente cantáridas para una meningitis tuberculosa ${ }^{61}$. Todos estos cuidados que se dispensaban en el Hospital Clínico de Madrid a estos enfermos crónicos e incurables son consistentes con los listados de recetas pedidos por el Hospital de Nuestra Señora del Carmen a la botica del Hospital de la Princesa, que veíamos al inicio de este apartado. Nos inclinamos a pensar que los enfermos de dicho hospital

\footnotetext{
55. Curso Académico de 1881-82, n. 50, Cap. 4, p. 54.

56. Curso Académico de 1881-82, n. 50, Cap. 2, p. 45 y Cap. 2, p. 25.

57. Curso Académico de 1881-82, n. 50, Cap. 3, p. 44.

58. Curso Académico de 1881-82, n. 50, Cap. 4, p. 54

59. Curso Académico de 1881-82, n. 50, Cap. 3, p. 27

60. Curso Académico de 1881-82, n. 50, Cap. 1, p. 84.

61. Curso Académico de 1881-82, n. 50, Cap. 1, p. 55.
} 
recibían un trato similar al recibido por los enfermos del Clínico. No es de extrañar, por tanto, que compartieran la descripción de los enfermos hecha por estos estudiantes de medicina:

«Adopta cualquier decúbito; gran demacración (...) ojos empañados y de mirada triste, voz débil [...] tiene color amarillento del rostro, mirada triste y facies característica de un padecimiento crónico» ${ }^{62}$.

\section{Conclusiones}

La asunción por el Estado del cuidado de los enfermos incurables tuvo importantes consecuencias en diversos ámbitos de la medicina. Produjo, en primer lugar, una serie de cambios en la definición del concepto «enfermedad incurable», cambios motivados por la necesidad de escoger un criterio que permitiese discriminar entre aquellos enfermos que podían ser acogidos en los hospitales para incurables y aquellos que no. Como hemos defendido en otros artículos, el principal efecto, a nivel teórico, consistió en la creación de definiciones locales del concepto de «enfermedad incurable», que se ajustasen y funcionasen en el contexto general de la realidad asistencial de cada una de las instituciones ${ }^{63}$. «Enfermedad incurable» se convertirá así en un concepto maleable, inclusivo, y relativo ${ }^{64}$. Esto sólo fue posible debido a la laxitud del concepto «enfermedad incurable», de ahí que los textos básicos para el tratamiento de las enfermedades de Charcot y Durand-Fardel, ambos traducidos al español, estuvieran profundamente condicionados por la práctica llevada a cabo en la Salpêtrière ${ }^{65}$. Fue la realidad asistencial la que dio contenido al concepto, y no al contrario, como podríamos haber esperado.

Pero siendo este un importante efecto de la medicalización, no fue, sin embargo, el más importante. Mayor fue el sufrido por los pacientes internados en estos hospitales, y que podemos cifrar, de forma resumida, en una falta absoluta de expectativas. El enfermo que era internado en uno de estos

\footnotetext{
62. Curso Académico de 1881-82, n. 50, Cap. 1, p. 80.

63. Zaragoza, n. 2.

64. Szabo, n. 5, p. 18.

65. Charcot, Jean Martin. Lecciones clínicas sobre las enfermedades de los viejos y las enfermedades crónicas. Madrid: Enrique Teodoro; 1883. Durand-Fardel, Max. Tratado práctico de las enfermedades crónicas. 3 vols. Madrid: Enrique Teodoro; 1877-1878.
} 
hospitales no sólo perdía la posibilidad de la curación de su enfermedad, sino que perdía también su vida, sin que por ello viera aliviados los males que sufría. Esta es la impresión que se extrae tras el estudio realizado, pero también de los testimonios privilegiados de Hauser o de Baroja, que en diversas ocasiones, sobre todo en El árbol de la ciencia ${ }^{66}$, nos aterra con las oscuras descripciones de la vida hospitalaria. Esta espera sin esperanza tal vez sea la condena mayor a la que estos incurables podían ser castigados, tal vez incluso mayor que vivir una vida de miseria en las calles de Madrid, arrastrando su dolor por las calles de Lavapiés o por el Rastro:

«Comprenez-vous, cher lecteur, un mot plus brutal que celui-là? C'et un arrêt, c'est une sentence! [...] Incurable... vous prononcez cela sans tressaillir? Ne comprenez-vous donc pas qu'il renferme tout un avenir de découragement, des jours lents et pleins de tortures» ${ }^{67}$.

66. Baroja, Pío. El árbol de la ciencia [1911]. Madrid: Caro Raggio / Cátedra; 2008.

67. Massé, Jules (1861). Trois maladies réputées incurables. Épilepsie-Dartres-Scrofules (Exemples de guérison). Paris: P. Brunet; 1861, p. 5. 


\section{ARTICLES}

Incurable disease in Spain during the 19th century. The Hospital para Hombres Incurables Nuestra Señora del Carmen

Juan Manuel Zaragoza..................................

1.--Introduction. 2.-Reform of charities. 3.-Nuestra Señora del Carmen Hospital for Incurable Men. 3.1.-Regulations for the management of Hospitals for Incurables. 3.2.-Requests for admission. 3.3.-Diseases and remedies. 3.4.-Prescriptions and poultices. 4.-Conclusions. 
ABSTRACT: This paper examines the State's assumption of medical care for patients with «permanent needs» in 19th century Spain. These patients were the incurably ill, the chronically ill and the elderly. This process is contextualized within the liberal reforms of the Spanish healthcare system in the reign of Isabel II (1833-1868). The goal of these reforms was the creation and consolidation of a national health system that would gradually replace the religious health charities. Healthcare reform became necessary due to the increase in migration that started in the 1830's and intensified in the 1850's. Traditional care networks formed by the family, local community and religious charities were no longer available to those who had left their village or town. In addition, many religious charities were bankrupted by the seizure of their properties in a programme of confiscation. Similar healthcare reform processes were taking place in the United Kingdom, France and Germany, among other European countries, and involved significant changes in the lives of patients, who became strictly controlled and medicalised. My aim was to identify changes in the patients' experience of illness through a case study of the living conditions of inmates at the Nuestra Señora del Carmen Hospital for Incurable Men, based in Madrid from 1852 to 1949. This was one of the institutions devoted to caring for patients with "permanent needs» and was under the direct control of the General State Administration. 